\title{
Sensory Evaluation of Nagpur Mandarin Powder Cookies using Fuzzy Logic
}

\author{
B. N. Patil", S. V. Gupta, S. B. Solanke and S. D. Deshmukh \\ Department of Agriculture Process Engineering, Dr. PDKV, \\ Akola-444001, M.S., India \\ *Corresponding author
}

Keywords

Sensory evaluation, Fuzzy logic,

Triplets, Similarity values

\section{Article Info}

Accepted:

15 July 2020

Available Online:

10 August 2020

\section{A B S T R A C T}

Nagpur mandarin powder cookies (S2) prepared from composite sample of wheat flour $(100 \mathrm{~g})$ and $10 \%$ Nagpur mandarian powder. Prepared Nagpur mandarin powder cookies (S2) was evaluated along with similar commercial food sample (coded as S1, S3) for their liking by trained panel members using standard fuzzy logic sensory technique. The developed samples were tested for their quality attributes as colour, flavour, texture and overall acceptability. The responses of the panel members were obtained in terms of not satisfactory, fair, medium, good and excellent. These quality attributes were considered as mathematical variable and based on these variables the fuzzy logic mathematical model was developed. As per output given by fuzzy logic model, the samples were ranked excellent, very good, good, satisfactory, fair and not satisfactory. On comparison of highest similarity values, their ranking was done as sample $S_{1}$ and $S_{3}>S_{2}$. Thus, it indicates that all three samples were preferred by judges. Also, the score of sample $S_{3}$ and $\mathrm{S}_{2}$ was very close under the category of "good". Therefore, present method of fortification by Nagpur mandarin powder cookies are similar to the commercial cookies and improve the sensorial and nutrition property of cookies.

\section{Introduction}

In today's world, the pace of life is fast and it has become virtually impossible to follow the three or four meal pattern that was traditionally accepted. Now the emphasis has shifted to more "snacks" in the meal pattern. It has been found to be more convenient to prepare a snack which can meet the nutritional needs of the family. Baking industry occupies an important position among Indian food processing industries. The spurt in the production of bakery products could be attributed to their advantages over other processed foods. Bakery products are ready to eat, convenient to use and posses satisfactory nutritional quality. India is the second largest producer of biscuits after USA. The biscuit industry in India comprise of organized and un-organized sectors. But one of the biggest challenges for product development is the acceptability by the consumers. Therefore, sensory test is required to predict the consumer acceptability and success of the product. In addition to this, satisfying the demands of the consumers is a 
major issue in order to succeed in promoting the consumption of functional products. For deciding the consumer choice towards the food products, sensory parameters followed by the nutritional properties are required to be considered. Due to this reason, sensory analysis of any developed food product is an important concern prior to supply the product in the market (Das, 2005; Routray and Mishra, 2011).

Sensory evaluation is an important tool in food industry. Fuzzy logic is an important tool by which imprecise data can be analyzed and important conclusions regarding acceptance, rejection, ranking, strong and weak attributes of food can be drawn (Shinde et al., 2014). In fuzzy modelling, linguistic variables (e.g., not satisfactory, good, excellent, etc.) are used for developing relationship between independent (e.g. color, flavor, texture, overall acceptance etc.) and dependent (e.g. acceptance, rejection, ranking, strong and weak attributes of food) variables (Das, 2005; Routray and Mishra, 2011).

Fuzzy sets used for analysis of sensory data instead of average scores to compare the sample's attribute (Lincklaen et al., 1989; Kavdir and Gayer, 2003). Zadeh (1965) introduced Fuzzy sets theory, which allows uncertain phenomena to be treated mathematically. Chen et al., (1988) developed a model for the analysis of sensory data. Zhang and Litchfield (1991) developed fuzzy comprehensive model for ranking of foods and developing new food products. Multiple experts are involved in subjective evaluation

Ranking of food samples and their quality attributes are based on triplets associated with sensory scales, triplets for sensory score, triplets for sensory score of quality attributes, triplets for relative weightage of quality attributes, triplets for overall sensory score, values of membership function of standard fuzzy scale, values of overall membership function of sensory scores on standard fuzzy scale, similarity values, quality attributes ranking in general.

Sensory evaluation comprises a set of techniques for accurate measurement of human responses to foods and minimizes the potentially biasing effects of brand identity and other information influences on the consumer perception. This method has been successfully applied for mango drinks (Jaya and Das, 2003), dahi powder (Routray and Mishra, 2011), instant green tea powder (Sinija and Mishra, 2011) and soy paneer (Uprit and Mishra, 2002). Millet-based bread (Singh et al., 2012), composite minor millet flour based RTE snack food (Shinde and pawar, 2016) and Kharodi (Solanke and Jaybhaye, 2018). So, the prepared Nagpur mandarian powder cookies are compared along with commercial cookies.

\section{Materials and Methods}

\section{Preparation of Nagpur mandarian powder cookies for sensory evaluation}

Nagpur mandarin powder cookies prepared from composite sample of wheat flour (100g) and $10 \%$ Nagpur mandarian powder in microwave oven. The process parameters were optimized in terms of Beating time (12.88 min $13 \mathrm{~min}$ ), Baking temperature $\left(213.98{ }^{\circ} \mathrm{C} \sim 210{ }^{\circ} \mathrm{C}\right)$ and Baking time (19.63 min $20 \mathrm{~min}$ ). The data obtained by sensory evaluation of Prepared Nagpur mandarin powder cookies (S2) was evaluated along with similar commercial food sample, S1Cookies marry, S3- local manufactured cookies)) for their liking by trained panel members using standard fuzzy logic sensory technique and Matlab software (Shinde et al., 2014.) Twenty-two judges from the faculty and research scholars of the Department of Agricultural Process Engineering, Dr. PDKV, 
Akola (10 males and 12 females in age range of 25 to 45) were chosen for the sensory appraisal of the final product Nagpur Mandarian powder cookies (Table 2), focused on good fitness, combined awareness and sensory preferences, capacity to focus and understand, and experience with bakery foods. In keeping with the chosen paint, taste, texture and overall acceptability (OAA) attributes for each sample panel members were questioned. Prior to sensory assessment, the judges became acquainted with the concepts of bakery commodity consistency attributes. Before research, two or three samples were demanded to be taken and the flavor settings were provided in the score sheet first.

The panellists were asked to indicate their preference for each sample based on the selected quality attributes of colour, flavour, texture and overall acceptability by giving tick $(\sqrt{ })$ mark to appropriate respective fuzzy scale factor for each of the quality attributes of the sample after evaluating the samples (Jaya and Das, 2003). They were asked to take two or three pieces of samples before testing them and give the score for flavour first in the score sheet. Also, they were advised to rinse their mouth with lukewarm water between the testing of each sensory character (Das, 2005; Sinija and Mishra, 2011) and between testing the consecutive samples (Jaya and Das, 2003).

The samples were rated as "Not satisfactory", "Fair", "Medium", "Good" and "Excellent". Judges were also instructed to give rank to quality attributes of cookies in general, by giving tick $(\sqrt{ })$ mark to the respective scale factors, viz. "Not at all important", "Somewhat important", "Important", "Highly important" and "Extremely important". The set of observations were analyzed using Fuzzy analysis of sensory scores. This method utilizes linguistic data obtained by sensory evaluation. Ranking of the Nagpur mandarian powder cookies samples was done by using triangular fuzzy membership distribution function. Sensory scores of the Nagpur mandarian powder cookies samples were obtained by using fuzzy scores given by the judges, which were converted to triplets and used for estimation of similarity values used for ranking of samples (Shinde et al., 2016).

\section{Fuzzy modeling of sensory data}

The major steps involved in the fuzzy modeling of sensory evaluation are (1) calculation of overall sensory scores of Nagpur mandarian powder cookies food samples in the form of triplets (Fig. 1); (2) estimation of membership function on standard fuzzy scale (Fig. 2); (3) computation of overall membership function on standard fuzzy scale (Fig. 3); (4) estimation of similarity values and ranking of the Nagpur mandarian powder cookies samples; and (5) quality attribute ranking of Nagpur mandarian powder cookies samples in general.

A program in MATLAB 7.0 software was developed for the calculation of all the above mentioned steps (Das, 2005; McGarrity, 2008).

\section{Triplets associated with five point sensory scale}

The obtained sensory scores were converted into a set of three numbers, called ,triplet" on five point scale. Nagpur mandarian powder cookies samples and quality attributes were assigned fuzzy membership on a five point sensory scale (Das, 2005). The distribution pattern of five point sensory scale is poor/not at all important, fair/somewhat important, medium/important, good/highly important and excellent/ extremely important as shown in Figure 1. 
Set of three numbers known as "triplet" is used to represent triangular membership function on five point scale where triangle ,abce represents membership function for poor/not at all important category, triangle , ade $^{\text {ce }}$ represents distribution function for fair/somewhat important category, etc. Table 1 shows 'triplets' associated with five point sensory scales. First number of the triplet denotes the value of abscissa at which the value of membership function is one. Second and third number of the triplet indicates the distance to left and right respectively of the first number where the membership function is zero (Routray and Mishra, 2011).

The triplet for a particular quality attribute of given sample can be obtained from the sum of sensory scores, triplets associated with five point sensory scale and the number of judges. For example, the colour attribute of a sample, when total number of judges were 20 and out of the total 20 judges, three judges gave „Fair ${ }^{\text {ee }}$ score, six judges gave the score as „Medium"e, fourteen gave „Good ${ }^{\text {"e }}$ and seven gave „Excellent ${ }^{\text {ee }}$ the triplets for the sensory scores of colour can be calculated by Eq. 1 .

$C s_{1}=\frac{0(0025)+1(252525)+3(502525)+14(752525)+2(100250)}{20}$

Triplets for each quality attribute of all the samples and quality attributes of Nagpur mandarian powder cookies samples in general were obtained as per eq. 1 . Similarly, from the calculated values of triplets for quality attributes of of Nagpur mandarian powder cookies samples in general, the triplets for relative weightage of quality attributes (QRel) were also calculated.

The Relative weightage of the quality attribute $^{\text {ee }}$ for color, flavor, taste and mouthfeel were defined as: $\mathrm{QCrel}=\mathrm{QC} /$ Qsum, flavor: QFrel $=\mathrm{QF} /$ Qsum, taste: QTrel = QT/Qsum and for mouthfeel: QMrel
= QS/ Qsum, where Qsum is the sum of first digit of triplets of all quality attributes in general.

Triplets for overall sensory scores of Nagpur mandarian powder cookies samples

The triplets for overall sensory scores of Nagpur mandarian powder cookies samples were calculated using eq. 2 , in which triplet for sensory score for each quality attribute was multiplied with the triplet for relative weightage of that particular attribute and the sum of resultant triplet values for all attributes was taken.

$\mathrm{S} 01=\mathrm{CS}_{1} \times \mathrm{QC}_{\mathrm{rel}}+\mathrm{FS}_{1} \times \mathrm{QF}_{\text {rel }}+\mathrm{TS}_{1} \times \mathrm{QT}_{\mathrm{rel}}+0 \mathrm{~S}_{1} \times \mathrm{QO}_{\text {rel }}$

Where, CS1, FS1, TS1 and MS1 represents the triplets corresponding to the colour, flavor, taste and mouthfeel of sample one and QCrel, QFrel, QTrel and QMrel denotes the triplets corresponding to the relative weightage of quality attributes of Nagpur mandarian powder cookies samples in general. Using similar equations the overall sensory scores for all samples were calculated. The multiplication of triplet $(\mathrm{a} b \mathrm{c})$ with triplet $(\mathrm{d} e$ f) was done by applying a rule as given in Eq. 3.

$(a b c) \times(d e f)=(a \times d a \times e+d \times b a \times f+d$ $\times c) \ldots . .(3)$

\section{Membership function for standard fuzzy scale}

The triplets obtained by Five Point scale are converted into Six Point sensory scale referred to as Standard Fuzzy scale. The triangular distribution pattern of sensory scales using symbols F1, F2, F3, F4, F5 and F6 is given in Figure 2. Membership function of each of the sensory scales follows triangular distribution pattern where 
maximum value of membership function is one.

The values of fuzzy membership function lie between 0 and10. Therefore, values of F1 through $\mathrm{F} 6$ are defined by a set of 10 numbers as given in Eq. 4.

$\mathrm{F} 1=(1,0.5,0,0,0,0,0,0,0,0)$

$\mathrm{F} 2=(0.5,1,1,0.5,0,0,0,0,0,0)$

$\mathrm{F} 3=(0,0,0.5,1,1,0.5,0,0,0,0)$

$\mathrm{F} 4=(0,0,0,0,0.5,1,1,0.5,0,0)$

$\mathrm{F} 5=(0,0,0,0,0,0,0.5,1,1,0.5)$

$\mathrm{F} 6=(0,0,0,0,0,0,0,0,0.5,1)$

\section{Overall membership function of sensory scores on standard fuzzy scale}

The overall quality of the Nagpur mandarian powder cookies samples was linked to the standard fuzzy scale. The overall quality, as expressed by a triplet $(a, b, c)$ was represented by a triangle $\mathrm{ABC}$, shown in Figure 3.

The graphical representation of membership function of a triplet $(a, b, c)$ is given in Figure 3 . The figure shows that for a triplet $(a, b, c)$, when the value of abscissa is $a$, value of membership function is 1 and when it is less than $a-b$ or greater than $a+c$, the value is 0 . For a given value of $x$ on abscissa, value of membership function $B x$ can be expressed by similar triangles as given in eq. 5 .

$B_{x}=\frac{x-(a-b)}{b}$ for $(a-b)<x<a$

$\mathrm{B}_{\mathrm{x}}=\frac{(\mathrm{a}+\mathrm{c})-\mathrm{x}}{\mathrm{c}}$ fora $<x<(\mathrm{a}+\mathrm{c})$

$B_{x}=0$ forx $<(a-b)$ and $x>(a+c)$

For overall sensory quality of each of the samples and for quality attributes of Nagpur mandarian powder cookies samples in general, the value of membership function $B x$ at $x=0,10,20,30,40,50,60,70,80,90$ and
100 were found out from eq. 5. This membership function value of samples and quality attributes in general on standard fuzzy scale was given as set of 10 numbers which are ,(maximum value of $B x$ at $0<x<10$ ), (maximum value of $B x$ at $10<x<20$ ), (maximum value of $B x$ at $20<x<30$ ), (maximum value of $B x$ at $30<x<40$ ), (maximum value of $B x$ at $40<x<50$ ), (maximum value of $B x$ at $50<x<60$ ), (maximum value of $B x$ at $60<x<70$ ), (maximum value of $B x$ at $70<x<80$ ), (maximum value of $B x$ at $80<x<90$ ), (maximum value of $B x$ at $90<x<100)^{\text {ee }}$.

\section{Similarity values and ranking of snack food samples}

After getting the B values for each sample and quality attribute in general on standard fuzzy scale as a set of 10 values, the similarity values for each triplet of samples and quality attributes were obtained by the eq. 6 (Sinija and Mishra, 2011).

$$
\mathrm{S}_{\mathrm{m}}(\mathrm{F}, \mathrm{B})=\frac{{\mathrm{F} \times \mathrm{B}^{\prime}}^{\prime}}{\operatorname{Max}\left(\mathrm{F} \times \mathrm{F}^{\prime} \text { andB } \times \mathrm{B}^{\prime}\right)}
$$

Where, $S m$ is the similarity value for the sample and quality attribute in general, $F \times B^{\prime}$ is the product of matrix $F$ with the transpose of matrix $B, F \times F^{\prime}$ is the product of matrix $F$ with the transpose of $F$ and $B \times B^{\prime}$ is the product of matrix $B$ with its transpose. For sample one similarity values will be $S m(F 1$, $B 1), S m(F 2, B 1), S m(F 3, B 1), S m(F 4, B 1)$, $S m(F 5, B 1)$ and $S m(F 6, B 1)$. The values were calculated using the rules of matrix multiplication.

Similarity values under the six categories of sensory scales were compared to find out the highest similarity value. The category corresponding to the highest similarity value was considered responsible for its quality. The overall quality of each of the samples 
was defined using above procedure. By combining the defined overall qualities of the samples as calculated by the above procedure, ranking of three samples and quality attributes in general was done by fuzzy comprehensive modeling (Zhang and Litchfield, 1991).

\section{Results and Discussion}

Jaya and Das, (2003) Stated that fuzzy logic can be applied to treat uncertain phenomena mathematically, i.e., expressing the degree of ambiguity in human thinking and relating it to a real number. The fuzzy logic technique converts the linguistic sensory responses obtained from the judges into numerical values which can be applied for comparison of similar products. The sensory scores as given by the judges have been shown in and Table 2.

\section{Triplets for sensory quality of RTE snack food sample}

Table 2 shows the sum of sensory scores according to preferences given by the judges for Nagpur mandarian powder cookies samples as S1, S2 and S3.

Triangular membership function distributions of sensory scales were given by "triplets", which are sets of three numbers. Calculation of sensory quality attributes of all Nagpur mandarian powder cookies samples in triplets form (Column 6 of Table 2) was done from (i) sum of sensory scores given by judges during sensory evaluation, (ii) triplets associated with the sensory scale (Table 1) and (iii) number of judges who gave tick mark under particular head on sensory scale (Table 2). The triplet for a particular quality attribute of given sample was obtained from the sum of sensory scores, triplets associated with five point sensory scale and the number of judges. The results of calculations of triplets for three samples under sensory evaluation are given in Table 2.

Triplets for importance of quality attributes in general and relative weightage

The sensory scores given by judges and the triplets for quality attributes in general of snack Nagpur mandarian powder cookies samples are given in Table 3. The triplets for individual preference to the importance of quality attributes of Nagpur mandarian powder cookies samples in general were calculated using the eq. 1 similar to triplets for three samples. Thus, the triplets for judges preference to importance of quality attributes, viz., colour (QC), flavour (QF), texture (QT) and overall acceptability (QO) were as given in Table 3.

It is necessary to bring the value of the first digit of overall sensory score between 0 and 100. In order to do this, the values of triplets for quality attributes in Table 3 were reduced by a factor 1/Qsum, where, Qsum is the sum of the first digit of all the triplets which was calculated (Singh et al., 2012).

Table.1 Triplets associated with five point sensory scale

\begin{tabular}{|l|l|l|l|l|l|}
\hline $\begin{array}{c}\text { Poor/Not at all } \\
\text { important }\end{array}$ & $\begin{array}{c}\text { Fair/Slightly } \\
\text { important }\end{array}$ & $\begin{array}{c}\text { Good/ } \\
\text { Important }\end{array}$ & $\begin{array}{c}\text { Very } \\
\text { good/Highly } \\
\text { important }\end{array}$ & $\begin{array}{c}\text { Excellent/ extremely } \\
\text { important }\end{array}$ \\
\hline 0 0 25 & 252525 & 502525 & 752525 & 100250 \\
\hline
\end{tabular}


Table.2 Sensory scores in terms of preference given by judges and corresponding triplets for sensory quality of cookies

\begin{tabular}{|c|c|c|c|c|c|c|}
\hline SQA & NS & $\mathbf{F}$ & $\mathbf{M}$ & $\mathbf{G}$ & $\mathbf{E}$ & Triplets for Sensory Quality of Samples \\
\hline \multicolumn{7}{|c|}{ Colour } \\
\hline $\mathbf{S}_{1}$ & 0 & 3 & 7 & 10 & 2 & $\mathrm{CS}_{1}=\left(\begin{array}{llll}63.50 & 25.00 & 22.73\end{array}\right)$ \\
\hline $\mathbf{S}_{2}$ & 0 & 3 & 8 & 8 & 3 & $\mathrm{CS}_{2}=\left(\begin{array}{llll}72.50 & 25.00 & 21.59\end{array}\right)$ \\
\hline $\mathbf{S}_{\mathbf{3}}$ & 0 & 4 & 7 & 9 & 2 & $\mathrm{CS}_{3}=\left(\begin{array}{ll}60.2325 .00 & 22.73\end{array}\right)$ \\
\hline \multicolumn{7}{|c|}{ Flavour } \\
\hline $\mathbf{S}_{1}$ & 0 & 1 & 2 & 11 & 8 & $\mathrm{FS}_{1}=(79.5525 .0015 .91)$ \\
\hline $\mathbf{S}_{2}$ & 0 & 2 & 5 & 9 & 6 & $\mathrm{FS}_{2}=\left(\begin{array}{l}71.5925 .0018 .18\end{array}\right)$ \\
\hline $\mathbf{S}_{3}$ & 0 & 3 & 3 & 10 & 6 & $\mathrm{FS}_{3}=(71.5925 .0018 .18)$ \\
\hline \multicolumn{7}{|c|}{ Texture } \\
\hline $\mathbf{S}_{1}$ & 0 & 0 & 5 & 12 & 5 & 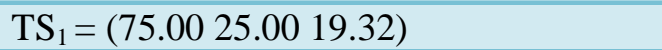 \\
\hline $\mathbf{S}_{2}$ & 1 & 2 & 8 & 8 & 3 & $\mathrm{TS}_{2}=\left(\begin{array}{l}61.36 \\
23.86\end{array} 21.59\right)$ \\
\hline $\mathbf{S}_{3}$ & 1 & 3 & 9 & 8 & 1 & $\mathrm{TS}_{3}=\left(\begin{array}{lll}55.68 & 23.86 & 23.86\end{array}\right)$ \\
\hline \multicolumn{7}{|l|}{ OAA } \\
\hline $\mathbf{S}_{1}$ & 0 & 2 & 5 & 10 & 5 & 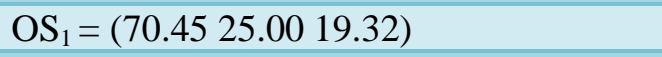 \\
\hline $\mathbf{S}_{2}$ & 1 & 3 & 6 & 8 & 4 & $\mathrm{OS}_{2}=\left(\begin{array}{lll}62.50 & 23.86 & 20.45\end{array}\right)$ \\
\hline $\mathbf{S}_{3}$ & 0 & 2 & 9 & 9 & 2 & $\mathrm{OS}_{3}=\left(\begin{array}{llll}62.50 & 25.00 & 22.73\end{array}\right)$ \\
\hline
\end{tabular}

Table.3 Sensory scores in terms of preference given by number of judges, triplets and relative weightage for quality attributes of cookies in general

\begin{tabular}{|c|c|c|c|c|c|c|c|}
\hline \multirow{2}{*}{$\begin{array}{c}\text { Quality } \\
\text { attributes }\end{array}$} & \multicolumn{5}{|c|}{ Sensory scale factors } & \multirow{2}{*}{$\begin{array}{c}\text { Triplets for } \\
\text { quality attributes }\end{array}$} & \multirow{2}{*}{$\begin{array}{l}\text { Triplets for } \\
\text { relative weightage }\end{array}$} \\
\hline & NI & SI & I & HI & EI & & \\
\hline Colour & 0 & 2 & 9 & 9 & 2 & $\mathrm{QC}=\left(\begin{array}{lll}68.75 & 27.50 & 25.00\end{array}\right)$ & $(0.20990 .08400 .0763)$ \\
\hline Flavour & 0 & 0 & 2 & 11 & 9 & $\mathrm{QF}=\left(\begin{array}{llll}91.25 & 27.50 & 16.25\end{array}\right)$ & $\left(\begin{array}{l}0.27860 .0840 \\
0.0496)\end{array}\right.$ \\
\hline Texture & 0 & 0 & 2 & 17 & 3 & $\mathrm{QT}=\left(\begin{array}{llll}83.75 & 27.50 & 23.75\end{array}\right)$ & 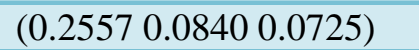 \\
\hline OAA & 0 & 0 & 5 & 11 & 6 & $\mathrm{QO}=\left(\begin{array}{l}73.75 \\
27.5020 .00\end{array}\right)$ & $(0.25570 .08400 .0611)$ \\
\hline
\end{tabular}

*NI - Not at all Important, SI - Somewhat Important, I - Important, HI - Highly Important, EI - Extremely Important

Table.4 Similarity values of cookies and their ranking

\begin{tabular}{|l|c|c|c|}
\hline Sensory scale & $\begin{array}{c}\text { Sample 1 } \\
\text { (Marry Cookies) }\end{array}$ & $\begin{array}{c}\text { Sample 2 } \\
\text { (Locally manufactured } \\
\text { Cookies) }\end{array}$ & $\begin{array}{c}\text { Sample 3 } \\
\text { (Cookies with Nagpur } \\
\text { mandarin powder) }\end{array}$ \\
\hline Poor & 0 & 0.004 & 0.008 \\
\hline Fair & 0.080 & 0.136 & 0.158 \\
\hline Medium & 0.347 & 0.461 & 0.486 \\
\hline Good & 0.647 & $\mathbf{0 . 7 1 6}$ & $\mathbf{0 . 7 1 8}$ \\
\hline Very good & $\mathbf{0 . 6 6 7}$ & 0.565 & 0.530 \\
\hline Excellent & 0.245 & 0.164 & 0.147 \\
\hline Ranking & I & III & II \\
\hline
\end{tabular}


Table.5 Similarity values of quality attributes of cookies in general

\begin{tabular}{|l|c|c|c|c|}
\hline Sensory scale & Colour & Flavour & Texture & $\begin{array}{c}\text { Overall } \\
\text { Acceptability }\end{array}$ \\
\hline Not at all Important & 0 & 0 & 0 & 0 \\
\hline Somewhat Important & 0 & 0 & 0 & 0 \\
\hline Important & 0.236 & 0 & 0.027 & 0.027 \\
\hline Very Important & 0.829 & 0.209 & 0.418 & 0.427 \\
\hline Highly Important & 0.743 & 0.864 & 0.971 & 0.983 \\
\hline Extremely Important & 0.152 & 0.639 & 0.484 & 0.477 \\
\hline Ranking & IV & III & II & I \\
\hline
\end{tabular}

Fig.1 Triplets associated with five point sensory scale

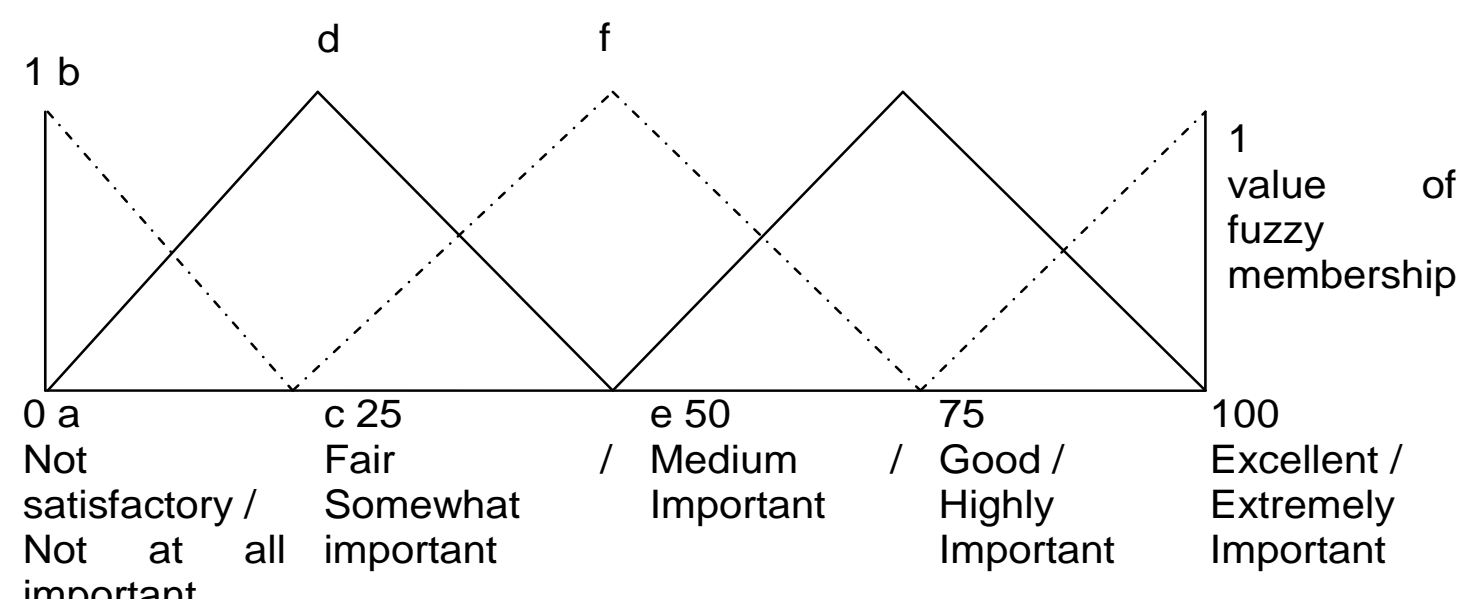

Fig.2 Standard fuzzy scale

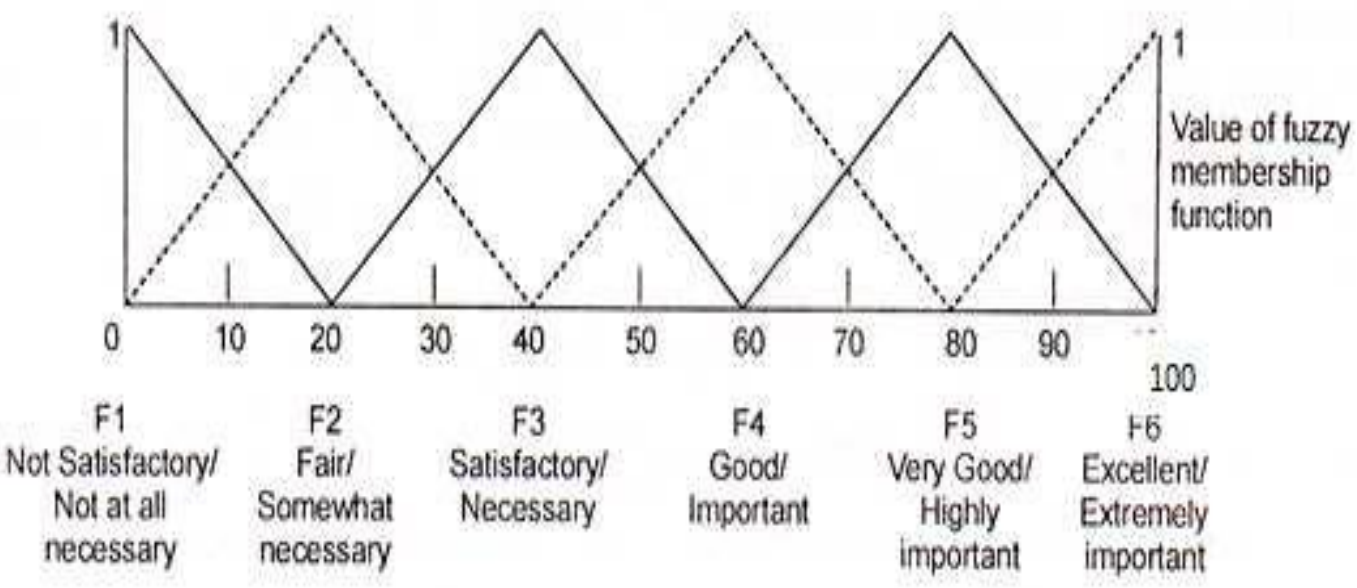


Fig.3 Graphical representation of triplet (a, b, c) and its membership function

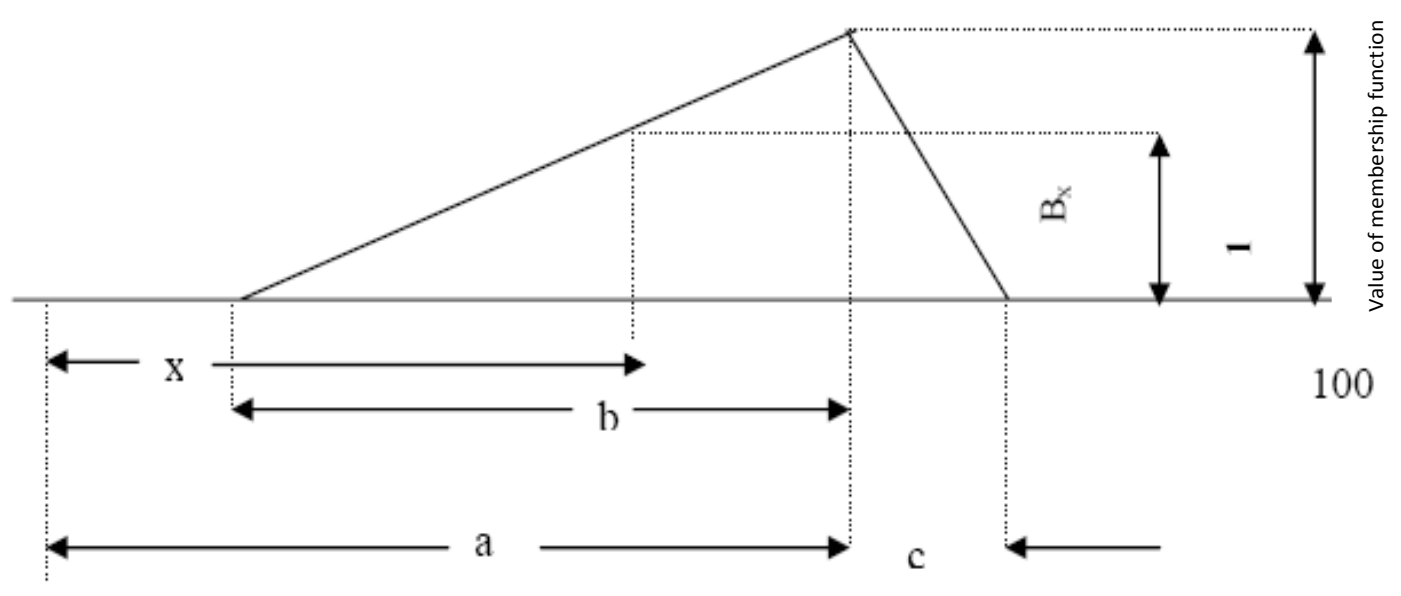

The values of triplets for sensory scores of prepared samples and relative weightages of the quality attributes were multiplied following the rule of multiplication of triplets mentioned in Eqn 7. The calculated values of triplets for overall sensory scores of Sample $\mathrm{S}_{1}\left(\mathrm{SOS}_{1}\right), \mathrm{S}_{2}\left(\mathrm{SOS}_{2}\right)$ and $\mathrm{S}_{3}\left(\mathrm{SOS}_{3}\right)$ are as follows:

$\mathrm{SOS}_{1}=(72.4800549 .1412237 .5434)$

$\mathrm{SOS}_{2}=(64.7423746 .0791136 .9405)$

$\mathrm{SOS}_{3}=(62.8122845 .7017737 .7559)(7)$

\section{Overall membership functions of sensory} scores on standard fuzzy scale

Six point sensory scale viz., poor/not at all necessary, fair/somewhat necessary, medium/necessary, good/important, very good/highly important, excellent /extremely important referred to as 'standard fuzzy scale' and designated as $\mathrm{F}_{1}, \mathrm{~F}_{2}, \mathrm{~F}_{3}, \mathrm{~F}_{4}, \mathrm{~F}_{5}$ and $\mathrm{F}_{6}$, respectively were used in the evaluation of sensory scores. Membership function values for the standard fuzzy scale have been presented in Eqn 3.86. Values of overall membership function of sensory scores of the samples on standard fuzzy scale, Bx were calculated using Eqn 8.
Overall membership functions of Sample $S_{1}$, $\mathrm{S}_{2}$ and $\mathrm{S}_{3}$ were calculated by using the values in Eqn 8 and the triplets obtained are given in Eqn 7.

$\mathrm{BS} 1=\left(\begin{array}{lll}0 & 0 & 0.135550 .339050 .542540 .74604\end{array}\right.$ $0.9495310 .79970 .533341)$

$\mathrm{BS} 2=\left(\begin{array}{lllll}0 & 0.0290 & 0.24603 & 0.46305 & 0.68006\end{array}\right.$ $\begin{array}{lllllll}0.89708 & 1 & 0.85767 & 0.58697 & 0.31626)\end{array}$

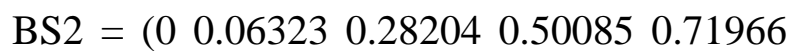
$0.938460 .809630 .544770 .2799)$

\section{Similarity values of cookies and their ranking}

Similarity values for cookies were calculated using the values of membership functions of standard fuzzy scale and overall membership function values of sensory scores (Eqn 8) by applying rules of matrix multiplication (Das, 2005; Sinija and Mishra, 2011). The ranking of samples was done on the basis of obtained similarity values under Poor $\left(\mathrm{F}_{1}\right)$, Fair $\left(\mathrm{F}_{2}\right)$, Medium $\left(\mathrm{F}_{3}\right)$, Good $\left(\mathrm{F}_{4}\right)$, Very good $\left(\mathrm{F}_{5}\right)$ and Excellent $\left(\mathrm{F}_{6}\right)$ categories. The similarity values for all the four samples under different scale factors are presented in Table 4. 
From the Table 4, it can be seen that, for sample $S_{1}$ having highest value obtained in the category "very good" is 0.667 and $S_{2}$ and $\mathrm{S}_{3}$ having similarity value lies in the category "good" i.e. 0.718 and 0.716 . On comparison of highest similarity values, their ranking was done as sample $S_{1}$ and $S_{3}>S_{2}$. Thus, it indicates that all three samples were preferred by judges. Also, the score of sample $S_{3}$ and $S_{2}$ was very close under the category of "good". Therefore, present method of fortification by Nagpur mandarin powder cookies are similar to the commercial cookies and improve the sensorial and nutrition property of cookies. Similar ranking under the category "very good" was obtained by Shinde et al., (2016) and Solanke et al., (2018) for "Kharodi".

\section{Quality ranking of Nagpur mandarin powder cookies}

The quality attributes of Nagpur mandarin powder cookies and other commercial sample in general were ranked by calculating similarity values under various scale factors. Values of membership functions of $\mathrm{F}_{1}, \mathrm{~F}_{2}, \mathrm{~F}_{3}$, $\mathrm{F}_{4}, \mathrm{~F}_{5}$ and $\mathrm{F}_{6}$ as given in Eqn 8 were used in the calculation of similarity values. The values of overall membership functions for sensory scores of the quality attributes viz., colour, flavour, texture and overall acceptability were calculated using the same procedure as described above. The similarity values of all the quality attributes are given in Table 5.

The results from Table 5 show that all quality attributes viz. colour, flavour, texture and overall acceptability can be considered as highly important for cookies in general. The overall acceptability (0.983) has highest quality attributes value followed by texture (0.971), flavour (0.864) and colour (0.829). The order of preference given by judges for quality attributes of cookies in general was overall acceptability (highly important) > texture (highly important) > colour (highly important) $>$ colour (highly important).

\section{References}

BIS, 1971. Guide for sensory evaluation of foods. Optimum requirements IS: 6273 (Parts-II). New Delhi: Bureau of Indian Standards.

Chen, S.M. 1988. A new approach to handling fuzzy decision making. IEEE Transactions on Systems, Man and Cybernetics, 18: 1012-1016.

Das, H. 2005. Food Processing Operations Analysis. Asian Books Pvt. Ltd. New Delhi, pp. 357-402.

Heidenreich, S., Jaros, D., Rohm, R., and Ziems, A. 2004and crispness of extruded rice crisps. J. of Texture Studies, 35:621-633.

Jaya, S., and Das, H. 2003. Sensory evaluation of mango drinks using fuzzy logic. Journal of Sensory Studies, 18:163-176.

Jayebhaye, R.V. 2012. Development of hot air puffing machine for preparation of millet based RTE snack food. Unpublished Ph. D thesis, Agricultural and Food Engineering Department, IIT, Kharagpur (W.B.)-721302, India.

Kavdir, I., and Gayer, D.E. 2003. Apple grading using Fuzzy logic. Turkish Journal of Agriculture, 27: 375-382.

Lincklaen, W., Westenberg, S.D., Jong, D.A., Meel, J.F.A., Quadt, E., and Duin, R.P.W. 1989. Fuzzy set theory applied to product classification by a sensory panel. Journal of Sensory Studies, 4(1): 55-72.

McGarrity, S. 2008. Introduction to objectoriented programming in MATLAB $®$, Matlab Digest. www.mathworks.com.

Oxford and IBH. Mazumder, P., Roopa, B.S., and Bhattacharya, S. 2007. Textural attributes of a model snack food at different moisture contents. Journal of 
Food Engineering, 79:511- 516.

Ranganna, S. 1987. Handbook of analysis and quality control for fruit and vegetables products, Tata McGraw Hill. Pp. 156 196.

Routray, W., and Mishra, H.N. 2011. Sensory evaluation of different drinks formulated from dahi (Indian yogurt) powder using fuzzy logic. Journal of Food Processing and Preservation, doi:10.1111/j.1745-4549.2011.00545.

Shinde, K.J., and Pardeshi, I.L. 2014. Fuzzy logic model for sensory evaluation of commercially available jam samples. Journal of Ready to Eat Food, 01(02): 78-84.

Shinde, K.J., Pardeshi, I.L., Pawar, S.G., and Gajakos, A.V. 2016. Applications of fuzzy logic technique in sensory evaluation of ready to eat foods. Journal of Ready to Eat Food, 03 (04): 45-50.

Singh, K.P., Mishra, A., and Mishra, H.N. 2012. Fuzzy analysis of sensory attributes of bread prepared from millet-based composite flours. $L W T$ Food Science and Technology, 48 (02): 276- 282.

Sinija, V.R., and Mishra, H.N. 2011. Fuzzy analysis of sensory data for quality evaluation and ranking of instant green tea powder and granules. Food and Bioprocess Technology, 4: 408-416.

Solanke, S.B., R.V. Jaybhaye and Jadhav, S.B. 2018. Sensory Evaluation of Pearl Millet based Snack Food (Kharodi) using Fuzzy Logic. In 2154.

Uprit, S., and Mishra, H.N. 2002. Fuzzy multi attribute decision making approach and comparison of soya fortified paneer. Journal of Sensory Studies, 17 (2): 163-176.

Zadeh, L. 1965. Fuzzy sets: Information and control, 8: 338-353.

Zhang, Q., and Litchfield, J.B. 1991. Applying fuzzy mathematics to product development and comparison. Food Technology, 45(7): 108-115.

\section{How to cite this article:}

Patil, B. N., S. V. Gupta, S. B. Solanke and Deshmukh, S. D. 2020. Sensory Evaluation of Nagpur Mandarin Powder Cookies using Fuzzy Logic. Int.J.Curr.Microbiol.App.Sci. 9(08): 1388-1398. doi: https://doi.org/10.20546/ijcmas.2020.908.158 